\title{
Belphégor
}

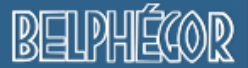

Littérature populaire et culture médiatique

19-2 | 2021

La civilisation du magazine

\section{Entre exégèse et fantasmagorie : Midi-Minuit fantastique et la naissance d'une cinéphilie alternative (1962-1970)}

Axel Hohnsbein

\section{(2) OpenEdition}

Journals

Édition électronique

URL : https://journals.openedition.org/belphegor/4272

DOI : $10.4000 /$ belphegor.4272

ISSN : 1499-7185

Éditeur

LPCM

Référence électronique

Axel Hohnsbein, « Entre exégèse et fantasmagorie : Midi-Minuit fantastique et la naissance d'une cinéphilie alternative (1962-1970) », Belphégor [En ligne], 19-2 | 2021, mis en ligne le 22 janvier 2022, consulté le 20 septembre 2022. URL : http://journals.openedition.org/belphegor/4272 ; DOI : https:// doi.org/10.4000/belphegor.4272

Ce document a été généré automatiquement le 20 septembre 2022.

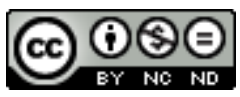

Creative Commons - Attribution - Pas d'Utilisation Commerciale - Pas de Modification 4.0 International - CC BY-NC-ND 4.0

https://creativecommons.org/licenses/by-nc-nd/4.0/ 


\title{
Entre exégèse et fantasmagorie : Midi-Minuit fantastique et la naissance d'une cinéphilie alternative (1962-1970)
}

\author{
Axel Hohnsbein
}

1 « Tout ce qu'édite Éric Losfeld sort de l'ordinaire en claquant les portes. » Cette formule aussi publicitaire que provocante ${ }^{1}$ caractérise bien Midi-Minuit fantastique, dont le nom fut choisi en hommage au Midi-Minuit, cinéma parisien spécialisé dans les thèmes « de l'étrange, du sexe ou de la violence - ou des trois à la fois ${ }^{2} . . . »$. Dès le titre, un programme est donc esquissé. Selon l'observation de Nicolas Stanzick, à qui l'on doit la très belle réédition du périodique ${ }^{3}$, Midi-Minuit fantastique est le premier périodique européen spécialisé dans le cinéma fantastique ${ }^{4}$. De 1962 à 1970, vingt-quatre numéros - dont certains doubles - se succèdent à un rythme irrégulier, à mesure que l'équipe fait face à des tensions internes, aux mutations du cinéma et de la société, la censure demeurant aux aguets. L'éviter n'est pas chose facile : Éric Losfeld, ami des surréalistes, est connu des censeurs pour avoir largement contribué à l'essor de l'édition érotique. La censure finira d'ailleurs par avoir raison de Midi-Minuit fantastique, alors même que le progressisme moral de mai 1968 se diffuse au sein de la société française.

2 Né de l'association improbable de deux étudiants, Michel Caen et Alain Le Bris, avec l'éditeur Éric Losfeld, l'artiste Jean Boullet - personnage aussi érudit que pittoresque et Jean-Claude Romer - explorateur infatigable de toutes les filmographies ${ }^{5}$, Midi-Minuit fantastique veut se spécialiser dans le cinéma de genre mais ne cesse d'opérer des pas de côté. Constitué d'étudiants démissionnaires, peintres, dessinateurs, journalistes, universitaires, cinéastes, libraires et éditeurs, le personnel de la revue est peu familier du journalisme professionnel. Le contenu visuel peut aussi paraître déroutant: photographies en tout genre, documents publicitaires, dessins, peintures, gravures, affiches, curiosa, BD, toutes ces images souvent rares et inédites sont souvent regroupées dans des cahiers iconographiques formant des «tunnels de trente pages ${ }^{6} »$. 
Quant au style d'écriture et à l'angle argumentatif, il varie énormément : Jean Boullet, Michel Caen, Roland Topor, Boris Vian, André Pieyre de Mandiargues, Eugène Ionesco, Félix Labisse, Bertrand Tavernier, Yves Boisset ou Tony Faivre côtoient des extraits de Jonathan Swift et Bram Stoker.

3 Vouloir définir le fantastique selon le périodique serait une erreur car cela reviendrait à forcer la cohérence de discours changeants : si l'aspect définitionnel est faible, c'est précisément parce que $M M F$ - selon l'acronyme qu'emploient ses rédacteurs - veut permettre au fantastique d'exister médiatiquement dans toute son hétérogénéité. Il s'agit d'ouvrir un canal de diffusion pour une iconographie alors difficile d'accès et de l'encadrer d'une collectivité de plumes - certes disparates. Comprendre l'hétérogénéité constitutive de MMF passe dès lors par la compréhension de son "énonciation éditoriale ", définie par Emmanuël Souchier comme "une configuration de supports matériels techniques et sémiotiques, de langages symboliques de natures distinctes, de pratiques de métiers et de pratiques sociales ${ }^{7} »$. Cet assemblage repose sur la connexion de deux réseaux interdépendants : réseau social (MMF devient un lieu d'échange entre rédacteurs, lecteurs, producteurs et promoteurs de cinéma fantastique) et réseau de contenus très variés unis par des thématiques obsédantes (érotisme, violence, monstres...), tous deux en expansion permanente. Évanghélia Stead et Hélène Védrine précisent qu'«un des avantages du terme réseau est qu'il souligne la dynamique relationnelle» unissant les personnes, mais aussi "les idées, les formes et leur action $»^{8}$. Étudier l'hétérogénéité même de l'assemblage de MMF permettra de montrer comment " une revue se constitue en réseau et compose son réseau ${ }^{9}$ ".

4 Notre objectif sera de montrer que la réussite de MMF ne réside pas dans ses qualités littéraires, analytiques ou esthétiques variables, mais dans la volonté structurante d'explorer et de développer les territoires d'un fantastique qui, s'il n'est jamais précisément défini, est envisagé comme un univers en croissance permanente, capable par exemple d'annexer les territoires de la science-fiction américaine ou japonaise et d'influencer les productions de la Nouvelle Vague. C'est-à-dire que, dans sa volonté de légitimer une cinéphilie alternative, $M M F$ devient à la fois la carte et le territoire. Partant d'une analyse des relations unissant le cœur du comité de rédaction, nous montrerons comment MMF a évolué de la revue vers le magazine, avant de montrer comment le périodique a contribué à créer un premier réseau d'échange autour du fantastique, pour achever sur l'étude du déploiement plus poétique que critique de ses illustrations.

\section{Une hydre à trois têtes}

5 Midi-Minuit fantastique paraît pour la première fois en mai 1962. Le récit des origines varie quelque peu selon les sources ${ }^{10}$ mais les faits principaux demeurent : étudiant en médecine devenu passionné de cinéma fantastique à la suite d'un séjour aux ÉtatsUnis ${ }^{11}$, Michel Caen envisage avec un ami du même âge, Alain Le Bris - lui-même sensibilisé au cinéma fantastique après un séjour en France -, de publier un livre sur le réalisateur phare de la Hammer, Terence Fisher. Fréquentant assidûment la librairie d'Éric Losfeld, Caen lui propose de publier l'ouvrage mais Losfeld lui suggère plutôt d'en faire le premier numéro d'une revue. Il lui conseille alors des collaborateurs, au premier rang desquels figure l'artiste Jean Boullet : collectionneur acharné de curiosa, homosexuel revendiqué et fervent adepte de chirurgie esthétique - il y recourt à 
plusieurs reprises ${ }^{12}$, il est plus âgé que Caen et Le Bris et connaît bien le cinéma fantastique d'avant-guerre ; autre suggestion de Losfeld : Jean-Claude Romer, appelé à devenir le spécialiste des filmographies de MMF. Un comité rédactionnel se forme ainsi rapidement, incluant aussi « l'écrivain Jacques Sternberg, Roger Cornaille le patron de la librairie Le Minotaure et Francis Lacassin, grand connaisseur de bandes dessinées ${ }^{13}$ ».

Dans son étude des poétiques journalistiques au xIX ${ }^{e}$ siècle, Marie-Ève Thérenty écrit que le journal est « une entreprise collective où s'expérimente la création du sens par fusion de voix plurielles et quelquefois discordantes ${ }^{14} »: M M F$ n'est certes pas un quotidien $\mathrm{du} \mathrm{xIX}^{\mathrm{e}}$ siècle mais cette analyse lui correspond bien, tant ce périodique apparaît comme le fruit de trois personnalités qui se complètent autant qu'elles se heurtent. Typiquement, MMF ne proposera pas d'éditorial avant son neuvième numéro, induisant un flou au sein duquel se manifestent au moins trois points de vue tantôt complémentaires, tantôt divergents: Michel Caen se rêve en chef d'une nouvelle pléiade, qui "pratiqu[erait] une permanente "défense et illustration du cinéma fantastique ${ }^{15}$ " "; bien qu'il fasse partie du comité éditorial, et qu'il édite la revue, Éric Losfeld peine quant à lui à s'identifier à la revue et se perçoit principalement comme un « garde-fou » pour des « jeunes gens » dont il craint qu'ils puissent céder à « l'apologie de la torture", instinctivement associée chez lui à la "fascination exercée par le fascisme ${ }^{16}{ }$; quant à Jean Boullet, très favorable à l'idée d'un cénacle dédié au cinéma fantastique, il ne conçoit pas d'autre chef de file que lui ${ }^{17}$. Se considérant comme le seul représentant vivant de la mémoire du cinéma fantastique ${ }^{18}$, Boullet possède quantité d'archives rares, ses «fabuleuses et démentielles collections ${ }^{19}$ » contribuant directement à l'identité graphique de la revue.

7 Les trois points de vue représentés par Caen, Losfeld et Boullet se côtoient ou se superposent tour à tour. Dans sa préface à la réédition du périodique, Nicolas Stanzick emploie une formule percutante pour caractériser MMF, dont « le ton est libertaire, les racines populaires, l'inspiration surréaliste ${ }^{20} »$. Cette dernière est rendue manifeste par l'investissement d'Éric Losfeld, dont la démarche montre qu'il existe une "véritable auctorialité de la part des imprimeurs-libraires ${ }^{21} »$ : ami d'André Breton, il peut solliciter un important réseau d'auteurs et artistes; en tant qu'éditeur de Positif, qui traverse sa phase surréaliste à cette période, Losfeld peut aussi garantir la présence ponctuelle de Robert Benayoun et Ado Kyrou dans les pages de MMF. Que ce soit par réelle conviction ou pour jouer un rôle de "garde-fou", l'éditeur contribue donc à rendre, par le recrutement de plumes et d'artistes relativement connus, la revue " prestigieuse dès son numéro $1^{22}$ ".

8 Le collectionneur et artiste Jean Boullet établit quant à lui de façon voyante le «ton libertaire» de la revue: son goût pour «tout ce qui n'[est] pas normal ${ }^{23}$ », son tempérament extravagant ${ }^{24}$ et l'esthétique même des dessins qu'il publie dans $M M F-$ Losfeld le considère comme une "espèce de sous-Cocteau ${ }^{25}$ " - font de lui un cousin plus ou moins proche des surréalistes. Surtout, la versatilité de sa plume s'avère essentielle à l'établissement du mythe de Midi-Minuit fantastique en tant que «revuemanifeste ${ }^{26} »$. C'est lui qui écrit le texte inaugural du numéro 1 , où la nuance n'a pas sa place :

Le mérite et l'audace de Michel Caen et d'Alain Le Bris ont été de vouloir sauver, à tout prix, dès maintenant, pendant qu'il en est temps encore, l'œuvre la plus agressivement insolite et violente du cinéma contemporain [celle de Terence Fisher]. Leur mérite est grand, alors que tant d'autres en sont encore à dresser la énième filmographie de John Ford, de Jean Renoir ou de René Clair [...]. 

la rédaction de Midi-Minuit fantastique et revendique le plaisir que provoque un cinéma insolite et violent - autant dire immoral. Boullet impose durablement le recours au style polémique. Typiquement, lorsque Jean-Paul Török écrit qu'« à une politique des auteurs nous préférons une politique des horreurs ${ }^{27} »$, il produit l'une des phrases iconiques de $M M F$, mais le sens résiste invinciblement à toute interprétation: si la politique des auteurs chère à François Truffaut s'appuie sur un riche corpus d'entretiens et d'articles, la politique des horreurs repose exclusivement sur la puissance d'une paronomase et le goût de la provocation.

10 À Losfeld le surréalisme, à Boullet la provocation libertaire : si l'on en revient à la formule de Nicolas Stanzick - et au risque de schématiser un peu le propos -, la responsabilité de donner un ancrage populaire à Midi-Minuit fantastique incombe dès lors à Michel Caen et Alain Le Bris. Par populaire, on ne parle pas tant d'origines sociales que de passion pour tout ce qui relève de la culture populaire: bandes dessinées, cinéma de quartier, roman feuilleton, etc. Refusant de concéder un droit d'aînesse à Jean Boullet, l'éditeur confie la revue à la jeunesse, permettant à Caen et Le Bris de «devenir les premiers rédacteurs en chef mineurs d'une revue interdite aux mineurs ${ }^{28} »$. Le mythe MMF se nourrit de cette situation paradoxale : des rédacteurs en chef mineurs, chargés de promouvoir une culture mineure interdite à la jeunesse, voilà qui transforme MMF en revue-manifeste annonciatrice de la contre-culture... même si, d'un point de vue pragmatique, Losfeld pensait plus simplement devoir confier le périodique à ces «deux jeunes gens, plus sérieux assurément que [Jean Boullet] ${ }^{29}$ ». Pétri de paradoxes et peu rompu aux méthodes journalistiques, Midi-Minuit fantastique trouve ainsi un premier équilibre aussi fascinant que fragile, qui ne manque pas d'évoluer dans le temps.

\section{Un Frankenstein de papier}

11 Marie-Ève Thérenty rappelle que la " matrice médiatique » repose sur quatre éléments centraux : la périodicité, la collectivité, la «rubricité» et l'actualité ${ }^{30}$. Midi-Minuit fantastique ne cessera de réfléchir aux usages de cette matrice. Dans un souci de clarté, nous délimiterons de façon un peu artificielle trois grandes périodes : la première $\left(\mathrm{n}^{\text {os }}\right.$ 1-6) correspond à l'alliance (et à son délitement) que nous venons de décrire entre Losfeld, Boullet, Caen et Le Bris. MMF privilégie alors des numéros thématiques centrés sur des créatures et films consacrés. La seconde période $\left(\mathrm{n}^{\text {os }} 7-13\right)$ correspond à un changement dans la direction du périodique, désormais dirigé par Michel Caen et JeanClaude Romer. MMF s'ouvre à l'actualité cinématographique et trouve sa place dans le paysage médiatique. La dernière période $\left(\mathrm{n}^{\mathrm{os}} 14-24\right)$ correspond à un changement de format et de maquette, le périodique s'ancrant dès lors pleinement dans une actualité $\mathrm{du}$ fantastique désormais bien visible.

Dans sa première période, Midi-Minuit fantastique se présente consciemment comme une revue. Jean Boullet utilise notamment le mot dès le seuil du premier numéro. Selon le Dictionnaire encyclopédique du livre, une revue parait à un rythme régulier (mensuel ou trimestriel le plus souvent) et réunit « soit des articles variés à l'intention d'un large public, soit au contraire des textes destinés à une catégorie particulière de lecteurs et consacrés à un domaine déterminé de connaissances ou d'intérêts ». MMF relève clairement de cette seconde catégorie, mais sa périodicité est alors assez aléatoire, tout 
comme sa pagination, qui demeure cependant conséquente, si bien que le format assez ramassé $(23 \times 15 \mathrm{~cm})$ qu'il utilise lui donne des airs de petit livre. Ce n'est pas rare à cette période: Louis Pauwels utilise par exemple un format assez proche pour ses revues Planète et Plexus. Visuellement parlant, MMF se situe pour ainsi dire à la jonction de la Troisième et de la Cinquième République. Les cahiers d'images, qui reproduisent quantité de photographies, affiches et matériel promotionnel en nuances de gris, lui confèrent une modernité typique des années 1960, tandis que le reste du maquettage le rattache à des traditions beaucoup plus anciennes : le texte est réparti sur une seule colonne, il s'ouvre souvent sur l'équivalent d'un bandeau et s'achève tout aussi souvent sur une variation de cul-de-lampe ; certains textes peuvent inclure des vignettes. Les images reproduites en dehors des cahiers ont ceci de très spécifique qu'elles sont reproduites dans des aplats de noir et de blanc séduisants et brutaux pouvant évoquer la gravure sur bois. La source de ces proto-bandeaux et culs-de-lampe n'est jamais donnée, il peut tout aussi bien s'agir de matériel promotionnel ou de dessins spécialement conçus pour l'occasion - Jean Boullet en signe un certain nombre ${ }^{31}$-, utilisés ici à des fins moins commerciales que décoratives et documentaires (perpétuer la mémoire du film et de son écho dans la société).

Visuellement parlant, le premier MMF est donc extrêmement éclectique : étalant volontiers les portraits photographiques de starlettes et de monstres contemporains, il s'ancre profondément dans un héritage médiatique ancien qui se manifeste fréquemment au détour des pages. Si Jean Boullet conspue «la retentissante imbécillité » de "l'ineffable Illustration ${ }^{32}$ » qui, dans un article de 1933, n'a visiblement rien compris à la réalisation des trucages de King Kong - les illustrations en sont reproduites dans le cahier d'images attenant -, Michel Caen décrit les Étrangleurs de Bombay comme " un très beau catalogue de tortures, un grand livre d'images dignes du regretté Journal des voyages que Fischer feuillette et nous dévoile avec une parfaite gratuitéz $^{33}$ ». En toute logique, MMF est friand des gravures du xIX siècle : le numéro 2 consacré aux «vamps fantastiques » reproduit par exemple en pleine page une gravure initialement publiée en 1882 dans un numéro de La Nature (fleuron de la vulgarisation scientifique encore publié à ce moment) et représentant un monstre de foire, la femme à trois têtes. Gustave Doré peut quant à lui aussi bien servir à décorer le début de la rubrique « Notes du mois ${ }^{34}$ » qu'être employé à des fins de provocation. N'ayant pas que des amis, MMF s'amuse par exemple à compiler quelques extraits des critiques et insultes qui lui sont adressées dans la presse ou directement par voie postale sous le titre de «La fée rageuse ${ }^{35}$ ». C'est une allusion directe à un personnage inventé par la comtesse de Ségur, initialement apparu dans le conte Ourson (1857), qui fait lui-même partie de l'ensemble des Nouveaux contes de fées pour les petits enfants illustrés par Gustave Doré. MMF prélève aussi une vignette de Doré pour la placer en tête de page : on y voit l'immonde fée rageuse à la tête d'un char en peau de crocodile traîné par un attelage de crapauds venimeux ${ }^{36}$. La revue répond à l'insulte verbale par une insulte picturale superbement choisie, ce qui lui permet par la même occasion de revendiquer son amour des monstres et de la culture fantastique dans un sens très élargi. Boullet est probablement la source principale de ces références appuyées à la culture visuelle du $\mathrm{XIX}^{\mathrm{e}}$ siècle et à l'univers des contes, auquel il rattache très vraisemblablement les monster movies, dont les créatures ne se sont, à son goût, «penché[e]s que sur de trop rares berceaux ${ }^{37} »$. 
14 La première incarnation de la revue connaît une fin prématurée après la publication du numéro 3, à la suite d'une dispute opposant Boullet aux rédacteurs en chef : persuadé qu'un passage du Gulliver de Jonathan Swift a directement inspiré la création de King Kong, Boullet demande au dessinateur Pierre Bailly de produire quelques illustrations de ce passage du roman en s'inspirant de photographies issues du film de Cooper et Schoedsack ${ }^{38}$... Ses jeunes collègues se montreront peu réceptifs à cette démarche, qui relève selon eux de la manipulation. Boullet claquera la porte à la suite de ce conflit. Le numéro 4-5, assez bref et consacré à Dracula, semble avoir été porté à bout de bras par Jean-Claude Romer, qui remplace Alain Le Bris au poste de corédacteur en chef pour le numéro 6: c'est le début de son "duo mythique ${ }^{39}$ » avec Michel Caen, qui signera le plus souvent seul les éditos de MMF.

15 S'ouvre alors une seconde période où les numéros ne sont plus centrés sur une œuvre ou une thématique unique : les couvertures annoncent fréquemment plusieurs sujets, quand elles ne se consacrent pas à l'« Actualité du cinéma fantastique » $\left(\mathrm{n}^{\circ} 7\right)$ ou à un "Tour du monde du fantastique " $\left(\mathrm{n}^{\circ} 9\right)$. L'évolution ne se situe pas tant ici dans le maquettage que dans le contenu. Globalement, MMF découvre et exploite mieux les vertus de la rubrique, qui selon Marie-Ève Thérenty «manifeste l'existence d'une temporalité plus pérenne» et "établit une continuité de lecture de numéro en numéro ${ }^{40} »$. Pour le quotidien de presse, elle ordonne l'actualité et la rend plus lisible ; pour la revue $M M F$, elle offre plutôt des repères visuels et montre que le périodique prend davantage conscience des nécessités qu'implique une temporalité médiatique. Toujours instable en termes de rythme de publication, MMF se montre un peu moins rétrospectif dans son approche et cherche à inclure, non sans humour, quelques passages obligés de la presse grand public. Les effets de rubriquage se remarquent ainsi davantage. "Ciné-guide", "previews ", top 10 des films de l'année, mais aussi une photo-mystère que les lecteurs doivent reconnaître, et surtout des mots croisés thématiques spécialement conçus par Jean-Claude Romer: la revue de niche se pare davantage des atours du magazine grand public ${ }^{41}$. MMF relègue par ailleurs un peu le texte d'analyse ou de description au profit des grands entretiens, s'ouvrant au dialogue direct avec les acteurs du cinéma fantastique contemporain et les responsables de festivals français et étrangers. L'équilibre même de la revue s'en trouve modifié, et cette découverte d'une nouvelle temporalité ne passe pas inaperçue aux yeux des lecteurs, qui s'expriment eux aussi depuis peu dans une rubrique «Correspondance »: «Dans votre dernier numéro, vous semblez changer de méthode et vous tourner vers le présent et l'avenir ; j'applaudis, mais je ne crois pas que pour cela il faille abandonner le travail archéologique que vous avez entrepris ${ }^{42}$...».

$M M F$ se doit donc de clarifier sa position et inaugure son premier édito dans le numéro 9. Michel Caen y affirme que la revue ne peut se «cantonner exclusivement à la fréquentation, très amicale, des loups-garous et vampires, sans risque de sclérose ${ }^{43}$ ». Affirmant la nécessité de nourrir la "quête de l'insolite au sein d'une apparente réalité $^{44}$ ", il ouvre définitivement Midi-Minuit fantastique aux influences de la Nouvelle Vague: Muriel de Resnais et Alphaville de Godard côtoient dès lors les films de la Hammer et les séries B de Roger Corman. Dans un entretien réalisé peu de temps avant son décès, Michel Caen reconnaît bien volontiers qu'il a "toujours été godardien », et que si « le midi-minuisme a puisé aux sources du surréalisme, et donc de Positif, [...] sa trajectoire rencontre assez rapidement celle des Cahiers $^{45} »$. Ce n'est pas un reniement mais un infléchissement : la provocation des débuts se nuance et Michel Caen redéfinit 
le positionnement médiatique de $M M F$, qui «ne pratiqu[e] finalement ni la politique des auteurs ni celle des horreurs " ${ }^{46}$. Le périodique navigue plutôt désormais dans un entre-deux qui ne cherche pas à tenir à tout prix un cap théorique ou une idéologique contraignante, et qui va s'attacher à valoriser le cinéma dès lors qu'il développe des accents fantastiques.

Le numéro 14 marque l'entrée du périodique dans sa troisième et dernière phase, celleci nettement plus dominée par l'actualité en tant qu'elle représente «le champ des intérêts partagés par le public à un moment donné ${ }^{47} »$. Nous sommes alors en 1966 : fort du succès de cette "très belle affaire commerciale ${ }^{48}$ ", Éric Losfeld décide - seul ? - de modifier la formule de la revue ${ }^{49}$, qui adopte alors le format agrandi typique de la presse magazine. Le maquettage est totalement revu: les couvertures arborent des photographies en couleurs et le texte est désormais réparti sur trois colonnes. L'image et le texte se mélangent volontiers. Les ordres de grandeur dans la typographie du titre sont inversés, «Midi-Minuit » se voyant réduit au profit d'un "fantastique » qui remplit toute la largeur de la couverture. Cela traduit l'évolution d'une politique éditoriale qui ne rend pas le périodique "moins midi-minuiste mais plus exactement midi-minuiste et caetera $a^{50}$ ». L'exploration du fantastique poursuit son expansion...

Cette nouvelle robe marque le début d'une période de crise pour la maquette, en proie à des ajustements permanents : chaque numéro propose un cahier graphique dans lequel un artiste est invité à présenter son œuvre, des nouvelles sont aussi proposées, un cahier de réclames en couleurs est inclus en fin de numéro, ainsi que la prépublication couleur de planches de bandes dessinées éditées par Losfeld - le lecteur tombe alors fréquemment nez-à-nez avec Barbarella ${ }^{51}$. Les lecteurs ne tarissent pas de commentaires sur ces changements et l'orientation plus marquée vers une pop culture ultracontemporaine, certains se montrant extrêmement sensibles à la disparition du regard rétrospectif qui faisait le propre de MMF :

Je ne connais votre revue que depuis peu, mais je me suis arrangée pour me procurer tous les numéros anciens et maintenant ma collection est complète. Mais je dois dire que mon plaisir augmentait à mesure que je remontais le fil de vos numéros. Des changements lents, mais certains, ont été effectués. Tout d'abord, la présentation qui pour être plus belle se rapproche plus d'une quelconque revue de cinéma. En ouvrant vos premiers numéros, nous avions l'impression de lire une revue "spéciale", c'était vieillot... ancien... délicieux. Maintenant nous avons [...] l'impression d'ouvrir un journal de Science-Fiction ${ }^{52}$.

19 Jean-Claude Bouyxou - lecteur de la première heure de $M M F^{53}$-, considère plus familièrement que « les choses devaient se gâter à partir du $\mathrm{n}^{\circ} 14$, avec [le] changement de format et [la] métamorphose en magazine plus ou moins chicos ${ }^{54} »$. Le commentaire est rude : défendre le fantastique alors qu'il est de plus en plus consommé et reconnu ne peut que passer par des changements importants de politique éditoriale - c'est un phénomène récurrent auquel se heurte actuellement le grand tenant de la presse spécialisée Mad Movies. Ces modifications ont aussi lieu peu de temps avant que Caen et Romer prennent en charge la programmation du Studio de l'Étoile ${ }^{55}$ en 1970: diminuant un peu plus l'attention portée à l'archéologie du fantastique, ils ancrent solidement MMF dans une actualité médiatique dont la maquette doit forcément rendre compte. Enfin, en homme de presse infatigable, Michel Caen est très probablement déjà en train de réfléchir aux deux futurs titres qu'il fondera en 1970 et 1975 : Zoom et L'Organe, toujours centrés sur la culture visuelle mais s'émancipant du fantastique pour augmenter la part photographique et érotique. 
20 En tant qu'œuvre close, MMF est donc le fruit d'influences extrêmement diverses: initialement imprégné d'un fantastique ancien mais toujours en quête de nouvelles influences, MMF est un ensemble composite mettant bout à bout des gravures du XIX siècle, des peintures surréalistes, des photographies de tournage, etc., son contenu reposant aussi bien sur la reproduction de trouvailles que sur des collaborations ponctuelles ou récurrentes. Ne cherchant pas à concilier ces diverses pratiques, les revendiquant toutes, MMF s'affiche ainsi comme un formidable Frankenstein de papier.

\section{L'élaboration d'un réseau fantastique}

21 En mouvement permanent, tant en ce qui concerne sa maquette que sa politique éditoriale, MMF se montre d'une cohérence remarquable dans sa volonté d'explorer les territoires du fantastique: exploration extensive des thématiques tout d'abord, qui pousse le périodique à rejeter la compartimentation des genres. Si les premiers numéros se concentrent sur les trésors du fantastique au cinéma (Dracula, Frankenstein, King Kong, Zaroff), les suivants ne cesseront d'élargir le champ d'exploration en intégrant les nuances fantastiques de la Nouvelle Vague, les innovations de Roman Polanski et la fantaisie pop de James Bond, en s'intéressant à la naissance du cinéma gore (voir l'accueil à la fois prudent et enthousiaste que réserve MMF à Blood Feast ${ }^{56}$ ), en accostant au Japon dans la seconde moitié de sa carrière, et en ouvrant finalement grande la porte de cette "succursale du fantastique » qu'est la science-fiction ${ }^{57}$.

Exploration géographique ensuite, amorcée après le départ de Jean Boullet, qui permet de communiquer directement avec ceux qui créent les œuvres. Cela passe d'abord par la correspondance. Les lettres d'auteurs prestigieux - dont le lecteur ignore qu'ils ont souvent été sollicités - sont toujours placées stratégiquement: la longue «Lettre de Christopher Lee ${ }^{58}$ » publiée dans le numéro 4-5 apparait, seule, sur deux pages ; dans un rectificatif qui augmente le rayonnement international de MMF, Schoedsack exprime son admiration pour la précision des études de la rédaction, même s'il écrit non sans ironie avoir été fort surpris de découvrir dans la revue que sa femme avait un passé de strip-teaseuse ${ }^{59}$; plus tard, ce seront par exemple William Castle ${ }^{60}$ ou Bela Lugosi Jr. ${ }^{61}$ Qui prendront (ou sembleront prendre) spontanément la plume pour écrire au périodique. Un réseau de correspondance se construit, le plus bel exemple apparaissant peut-être dans le numéro 10-11, lorsqu'un lecteur de la revue aide la rédaction à identifier Peter Cushing sur une image en joignant à sa lettre la réponse personnelle que l'acteur a bien voulu lui envoyer ${ }^{62}$. De tels échanges permettent de voir le réseau en train de s'établir, les réalisateurs, rédacteurs et simples amateurs ayant encore la possibilité d'échanger directement les uns avec les autres, le monde du cinéma fantastique n'étant pas encore aussi sociologiquement et professionnellement structuré que celui du cinéma traditionnel. Les intermédiaires tels que les attachés de presse sont d'ailleurs introuvables dans les pages du périodique.

Par la suite, MMF se dotera de correspondants officiels (le plus présent demeurant Raymond Durgnat pour l'Angleterre) et ira directement à la rencontre des professionnels du milieu: déplacements en Belgique, en Espagne, en Italie, en Angleterre, pour assister à des festivals, et pour rencontrer ceux qui font le cinéma fantastique. La nouveauté de la démarche apparaît cette fois dans la réaction des interviewés. Lors de son entretien avec $M M F$, c'est Riccardo Freda qui pose la première 
question: "on m'a prié de vous demander pourquoi vous vous intéressez exclusivement au genre fantastique ${ }^{63} \%$. On mesure ici à quel point l'existence du journalisme spécialisé ne va pas de soi, un réalisateur expert de la série B italienne tel que Freda pouvant être amené à demander des éclaircissements quant aux motivations du périodique. Outre ces échanges en cours de codification, les questions posées par les rédacteurs visent très fréquemment à cartographier les territoires du fantastique en tenant compte des affinités artistiques: Freda est appelé à se prononcer sur Terence Fisher ${ }^{64}$; on demande à Edgar G. Ulmer ce qu'il pense de Val Lewton et des films de Tarzan $^{65}$; à Roger Corman, ce qu'il pense de Nicholas Roeg ${ }^{66}$; à Fisher, s'il a vu Nosferatu ( «non ), Vampyr («dans l'ensemble, je suis plutôt contre ${ }^{67}$ ) ou Le Voyeur ("C'est horrible $\left.{ }^{68} »\right)$; à Tourneur, ce qu'il pense des adaptations de Frankenstein («Très mauvais. C'est le genre de choses que je déteste ${ }^{69} »$ ). L'aspect souvent péremptoire et cruellement sincère des réponses ne reflète pas tant l'amateurisme de l'interview que la liberté de propos dont jouissent des réalisateurs encore peu connus car considérés comme relevant du cinéma d'exploitation, un cinéma que l'on se représente encore majoritairement comme un produit à consommer mais pas à étudier.

Le périodique lui-même travaille à l'élargissement de son lectorat à mesure que sa forme évolue : une étude précise des modes de distribution et d'affichage publicitaire dont bénéficie $M M F$ demande à être menée afin de mieux mesurer le changement de palier qu'a pu représenter le passage de la forme revue à la forme magazine. La rubrique correspondance n'en dessine pas moins un axe Paris-province de plus en plus marqué - que nous n'avons malheureusement pas la place d'évoquer ici -, tandis que les sources de contenu visuel s'adaptent au succès grandissant du périodique : à l'origine, $M M F$ veut très clairement compiler et rendre disponibles des documents rares (disparus ou oubliés) que seuls quelques collectionneurs possèdent; dans ses derniers numéros, le périodique propose plutôt des photographies d'œuvres contemporaines vraisemblablement obtenues auprès des distributeurs. Contentons-nous ici de dire que, en devenant un espace de centralisation de la parole et des relations entre acteurs, rédacteurs et admirateurs, MMF se situe d'emblée à l'épicentre d'un réseau fantastique appelé à s'élargir considérablement par la suite, à se structurer, et à perdurer.

\section{Poétique de l'imagerie fantastique}

25 La masse des images (d'acteurs, d'actrices, de monstres anthropomorphes et d'animaux fantastiques), qui conservent tout leur impact aujourd'hui ${ }^{70}$, est une autre manifestation de l'intention des rédacteurs : au-delà même de la revendication d'un droit à exister pour le fantastique et ses amateurs, il s'agit de diffuser l'imagerie fantastique, tout en proposant un catalogue visant à satisfaire le(s) désir(s) du lectorat. En termes de proportion, l'image peut donc logiquement occuper une page complète.

Le désir du lecteur peut en premier lieu être de nature érotique, et nombre de textes portent la marque d'un enthousiasme pour des corps sexuellement désirables. Michel Caen considère que " [1]e lecteur de Midi-Minuit [est], par définition, érotomane averti lorsqu'il n'est pas érotologue ${ }^{71} »$. Il est donc fort possible que certaines images soient sélectionnées pour leur attrait masturbatoire : l'évolution même des couvertures à compter du numéro 14 montre d'ailleurs que MMF cherche de plus en plus à attirer le public de titres tels que Lui. L'imagerie de MMF associe cependant de façon récurrente l'érotisme à la violence ou à la monstruosité, les textes ne se risquant certes jamais à 
évoquer les fétichismes sexuels qui pourraient y être associés : contrairement à Jean Boullet, qui a toujours pris plaisir à revendiquer son homosexualité tout en affirmant des goûts esthétiques déviants, les jeunes rédacteurs manifestent quant à eux un appétit sexuel férocement hétéronormé, les jolies filles qui arborent une "poitrine démentielle " et "incroyablement décolletée ${ }^{72}$ " récoltant toujours les plus hauts suffrages - quitte à sombrer sur la fin dans le mauvais goût ${ }^{73}$. Dès lors, évoquer certaines perversions pénalement répréhensibles telles que la zoophilie (la jolie femme-singe, femme-araignée, femme-panthère ...) ou la nécrophilie (la jolie vampire) - toutes édulcorées car passées au prisme de la fiction cinématographique - manifeste plutôt la volonté voyante de rompre les digues morales de la France du général de Gaulle, tout en permettant plus simplement au lecteur de se rincer l'œil, le tout en évitant d'encourager les pratiques homosexuelles - ce qui vaudrait une interdiction de paraître instantanée ${ }^{74}$. Périlleux exercice d'équilibrisme, qui ne fonctionnera d'ailleurs pas, puisque MMF sera finalement interdit.

S'amuser des tabous, de façon finalement assez innocente mais néanmoins provocante dans le contexte français d'avant 1968, fait donc partie de la politique éditoriale de Midi-Minuit fantastique. Il s'agit même d'un pacte de lecture qui s'affiche dès la couverture du premier numéro, que Nicolas Stanzick présente ainsi :

Il s'agit d'une photo de plateau de La Nuit du loup-garou (Terence Fisher, 1961) : Oliver Reed y étrangle une jeune femme, dont on ne sait si elle est en train de jouir ou de mourir... C'est une photo "impossible" puisque dans le film, la belle violentée n'est autre que la mère du loup-garou, morte en le mettant au monde. Cette couverture est donc une sorte d'appel subliminal lancé aux spectateurs qui ont vu le film, sorti six mois plus tôt, seuls à même d'en comprendre la provocation incestueuse. C'est l'acte fondateur d'une communauté du regard, la naissance de la cinéphilie fantastique qu'on pourrait définir comme suit : le goût des mythes alliés à celui de la subversion ${ }^{75}$.

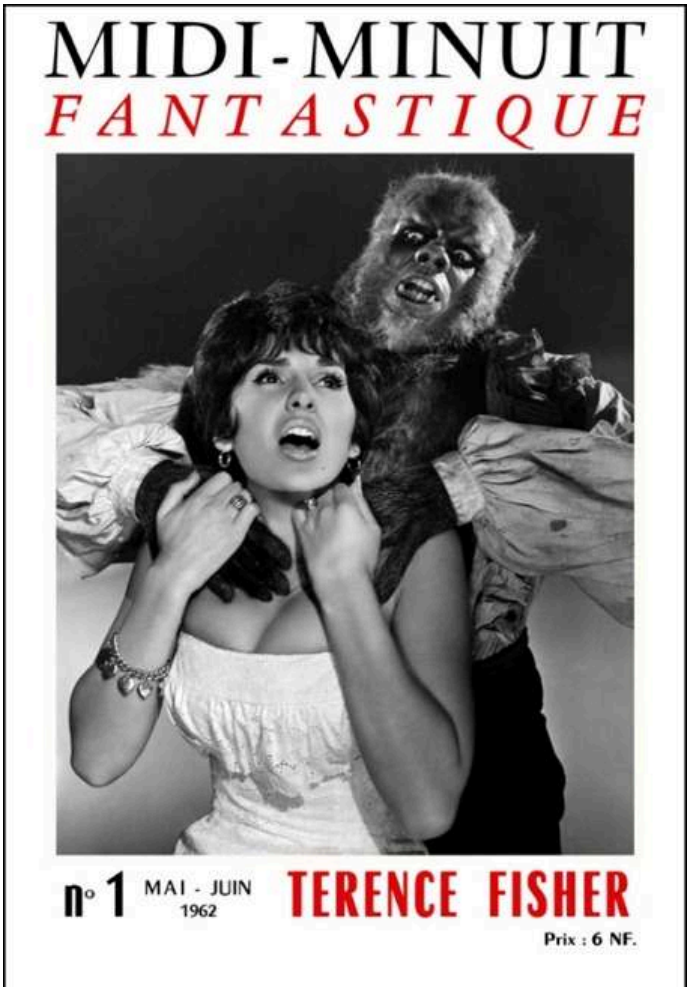

Couverture de Midi-Minuit fantastique, nº 1. Source : https://midiminuitfantastique.com/lintegrale-dunerevue-mythique/ 
L'image devient obscène une fois le contexte dévoilé : comptant sur l'ignorance totale de la censure en la matière, MMF dissimule brillamment ce pacte de lecture. Mieux encore : en valorisant d'emblée une image qui n'existe pas dans le film, MMF affirme la puissance d'évocation de l'imagerie fantastique, indépendamment de la qualité de l'œuvre dont elle est prélevée. Peut-être est-ce pour cette raison que Jean Boullet, malgré (ou plutôt grâce à) sa mythomanie invétérée, a tant apporté à la revue. Éric Losfeld raconte que, s'appuyant sur ses souvenirs du Masque de Fu Manchu ${ }^{76}$, Boullet pouvait réécrire des séquences entières : quand le film put enfin être vu, «ce fut un éclat de rire général ", et Losfeld de conclure que son "manque de sérieux » fut condamné "sans égard pour la poésie de ses mensonges, pour la création qu'ils représentaient ${ }^{77}$ ». Pourtant, les cahiers d'images que déroule MMF ne font rien d'autres que cela: inviter le lecteur à imaginer ses propres scénarios en créant un réseau d'images. Nicolas Stanzick évoque une "vraie dramaturgie des cahiers photo ${ }^{78}$ ", qui se nourrit de la difficulté même que l'on peut alors éprouver à voir ces films en France : l'image imprimée représente un objet de désir érotique/cinéphilique et une preuve unique de l'existence de l'œuvre, et elle entre simultanément en résonnance avec d'autres images de films tout aussi méconnus. Le réseau des thématiques (galeries de monstres $^{79}$, de femmes fleurs ${ }^{80}$, cahier consacré à Barbara Steele ${ }^{81} \ldots$ ) et des correspondances graphiques fait perdre à l'image une partie de sa valeur documentaire ou de preuve («ce film existe ») pour favoriser la rêverie fantasmagorique. L'exemple le plus volontairement visible réside dans les pages sobrement intitulées "Rencontres »: deux images se côtoient, la légende signalant simplement leur origine. Souvent, la similitude de la mise en scène saute aux yeux; parfois, elle trouble plus qu'elle ne clarifie l'interprétation, comme cette "rencontre » dans le numéro 3 entre un King Kong attaché les bras en croix et le Christ crucifié de Léon Bonnat.

Au-delà même de ces associations explicites, la totalité des images de MMF s'inscrit dans cette logique de rencontre. Par la mise en scène des images et la quête permanente d'échos tantôt évidents, tantôt inattendus, Midi-Minuit fantastique finit là encore par trouver efficacement sa place au sein du catalogue du Losfeld ami des surréalistes. Le périodique développe une imagerie qui déborde le cadre du cinéma et qui, par sa profusion et son défilement permanent, sature la mémoire du lecteur, suggérant diverses combinaisons et surimpressions, et provoquant in fine la cristallisation des représentations $d u$ fantastique dans l'inconscient collectif du lectorat. Cette démarche dépasse le seul cadre de la cinéphilie : à ses débuts, MMF se signale effectivement par sa difficulté à s'affranchir de l'approche descriptive ${ }^{82}$ et opte d'emblée pour des analyses relevant de ce que Nicolas Stanzick nomme la « cinéphilie littéraire ${ }^{83} »$. Cette approche permet la multiplication des canaux non pas de l'interprétation mais de l'imagination, permettant à cette dernière de s'emparer indifféremment d'objets textuels ou picturaux, imprimés ou filmiques, pour les unir au cours d'un feuilletage qui ne peut qu'inviter à la rêverie. MMF ne reniera jamais cette démarche littéraire. Les numéros 23 et 24 , qui seront les derniers publiés, constituent même sous certains aspects une ultime revendication de la légitimité de cette démarche : majoritairement occupés par Jean Rollin - qui a par exemple dirigé Brigitte Lahaie dans Fascination (1979) -, ces deux numéros ne traitent pas du cinéma éroticofantastique du réalisateur, qui figure plutôt ici en sa qualité d'auteur de deux textes fleuves consacrés à l'étude des œuvres littéraires et adaptations de Gaston Leroux. 


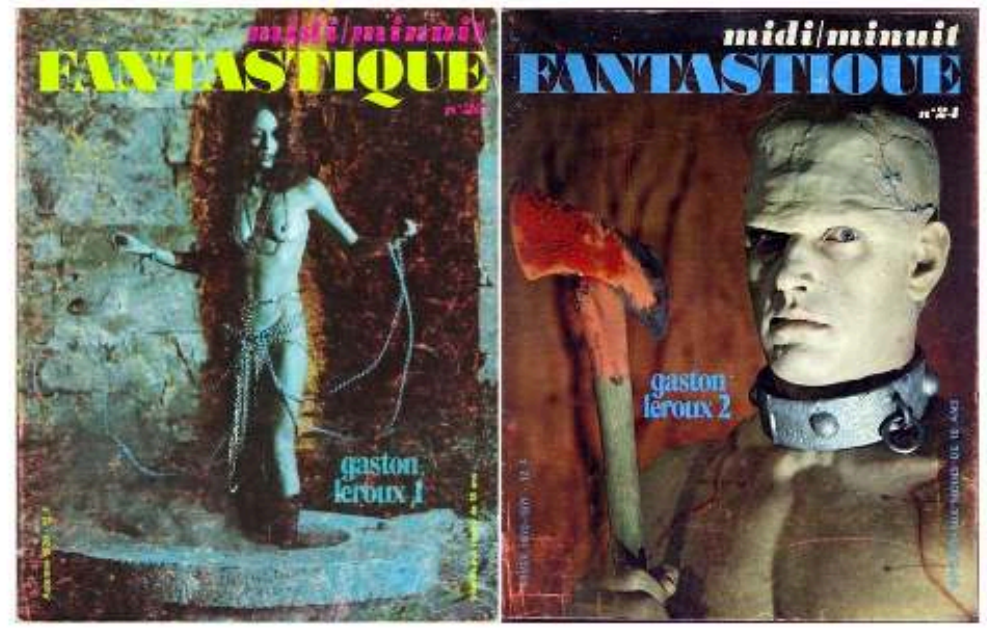

Couvertures des numéros 23 et 24 de Midi-Minuit fantastique.

d'un film de Rollin et d'un Frankenstein de la Hammer. L'association texte/image contrevient directement aux pratiques médiatiques traditionnelles, qui exigent que l'image corresponde au texte qui l'accompagne - a fortiori sur la couverture d'un numéro. La démarche ne manque pas d'inventivité - et d'humour. Parce que Leroux est un auteur aimé de Jean Rollin, il devient automatiquement objet d'intérêt pour la revue. Au lecteur en revanche d'interpréter à sa guise la relation unissant l'image et le texte, totalement cryptique tant qu'il n'a pas ouvert le numéro. On tient là un exemple de cette souplesse éditoriale dans l'usage de l'illustration, qui joue aussi bien sur des associations d'idées saugrenues que sur l'établissement de solides corpus thématiques.

Enfin, en dépit de l'immensité de son réseau, le cœur même de cette imagerie fantastique est aisé à localiser : Michel Caen précise volontiers dans ses interviews tardives qu' " une des raisons pour lesquelles [il a] voulu faire MMF était d'approcher Barbara Steele dont [il] étai[ $t$ ] follement amoureux ${ }^{84} »$. L'actrice italienne, qui a marqué l'histoire du cinéma pour son rôle de sorcière dans Le Masque du démon de Mario Bava, est effectivement omniprésente dans les pages du périodique, dont elle ornera la couverture par deux fois. Michel Caen devra attendre le numéro 12 avant de pouvoir enfin approcher cette femme dont il est tombé amoureux par images interposées. Ce lent cheminement s'achèvera de façon aussi poétique que fantastique : la rencontre de l'homme de presse et de l'actrice donnera lieu à une relation passionnelle ${ }^{85}$, puis l'amitié demeurera et Michel Caen tombera amoureux d'une autre femme, Geneviève Colange, lectrice et future collaboratrice de la revue, dont il partagera la vie jusqu'à son décès en 2014. Rarement la passion de l'image aura aussi directement influencé le destin amoureux d'un homme.

L'impact de Midi-Minuit fantastique est évident. Culturellement, le périodique a permis au cinéma fantastique d'exister tout d'abord en tant que maillon reliant le surréalisme et les arts populaires, puis en tant que genre cinématographique acceptable même aux yeux des grandes revues françaises de cinéma. Sociologiquement, il a permis de fédérer la communauté du fantastique à diverses échelles : parisienne tout d'abord, le centre de gravité se situant à la librairie du Terrain vague, au cinéma Midi-Minuit puis au Studio 
de l'Étoile ; internationale ensuite, le périodique ayant été le premier à jeter des ponts dans toute l'Europe, puis aux États-Unis et, enfin, en Asie ; nationale enfin, car une fois la correspondance établie, celle-ci ne s'arrêtera plus, témoignant du fossé séparant Paris de la province en termes de visibilité du fantastique, mais témoignant aussi de la reconnaissance éprouvée par des lecteurs soudainement projetés au cœur de la fabrique du fantastique cinématographique.

Médiatiquement, MMF donnera naissance à toute une lignée de fanzines, dont certains deviendront des magazines professionnels que l'on trouve encore en kiosque : JeanPierre Putters, fondateur de Mad Movies, est un fervent collectionneur des numéros de $M M F^{86}$; Alain Schlokoff, dont MMF publiera une lettre dans son numéro 21, fondera L'Écran fantastique; Christophe Gans, réalisateur et membre fondateur de Starfix, avoue bien volontiers que $M M F$, qu'il a lu dès ses dix ans, est sa « revue favorite de tous les temps ${ }^{87}$ ». À tout point de vue, Midi-Minuit fantastique constitue le socle fondamental de la cinéphilie fantastique française, tant sur le plan de sa consommation que de sa structuration médiatique.

\section{NOTES}

1. On la trouve par exemple en deuxième de couverture du $n^{\circ} 21$ de Midi-Minuit fantastique.

2. Jean-Claude Romer, "Vive le "Midi-Minuit" ", DeVilDead.com, lettre manuscrite datée du 14 avril 2000, [en ligne], consulté le 21/12/2020, URL: http://www.devildead.com/dossier/21souvenirs-de-midi-minuit-fantastique/17-vive-le-midi-minuit.

3. Ces ouvrages étant plus faciles à consulter que la série originale, nous renvoyons aux pages de la réédition. Cette étude tient compte $d u$ fait que la réédition diffère parfois des numéros originaux, qui ont été consultés lors de la rédaction de cet article. Nous évoquons plus longuement les propriétés remarquables de cette réédition sur notre carnet de recherche $L a$ Science en mouvement [En ligne], URL : https://lasciem.hypotheses.org/1450.

4. Voir la quatrième de couverture du premier volume de la réédition : Nicolas Stanzick et Michel Caen, Midi-Minuit fantastique, Pertuis/Aix-en-Provence, 2014-en cours (trois des quatre volumes sont parus chez Rouge Profond).

5. Dernier pilier de Midi-Minuit fantastique, Jean-Claude Romer est malheureusement décédé le 8 mai 2021. Nicolas Stanzick lui rend hommage sur le site consacré à la réédition du périodique, Midi-Minuit fantastique [En ligne], URL: https://midiminuitfantastique.com/2021/05/10/jeanclaude-romer-1933-2021/.

6. Stéphane du Mesnildot, «Midi-Minuit fantastique. L'école des monstres. Entretien avec Michel Caen et Nicolas Stanzick », Cahiers du cinéma, mars 2014, p. 84.

7. Emmanuël Souchier, «Formes et pouvoirs de l'énonciation éditoriale", Communications et langages, $\mathrm{n}^{\circ} 154,2007, \mathrm{p} .34$.

8. Évanghélia Stead et Hélène Védrine (dir.), L'Europe des revues II (1860-1930). Réseaux et circulations des modèles, Paris, Presses de l'université Paris-Sorbonne, 2018, p. 12.

9. Ibid., p. 15.

10. Voir Éric Losfeld, Endetté comme une mule, Auch, Tristram, 2017, p. 147-151; M. Caen, MMF, op. cit, vol. 1, p. 8, et vol. 2, p. 397-398 ; S. du Mesnildot, op. cit., p. 82.

11. S. du Mesnildot, id. 
12. Voir Denis Chollet, Jean Boullet, le précurseur, Nice, FEEL, 1999 ; et N. Stanzick, « Dracula et le montreur d'ombres ", MMF, op. cit, vol. 2, p. 361-390.

13. S. du Mesnildot, op. cit., p. 84.

14. M.-È. Thérenty, La Littérature au quotidien, Paris, Seuil, 2007, p. 62.

15. Michel Caen, « Midi-Minuit historique », MMF, op. cit., vol. 2, p. 397.

16. É. Losfeld, op. cit., p. 148.

17. Voir N. Stanzick., MMF, op. cit., vol. 2, p. 365.

18. "J'ai la chance d'être de ceux qui connaissent tous, je dis bien tous, les films qui précèdent dans le temps les sombres héros de la période pré-fishérienne et leurs différentes versions » $\mathrm{J}$. Boullet, « Terence Fisher et la permanence des mythes », MMF, op. cit., vol. 1, p. 45).

19. Cité par N. Stanzick, « Midi-Minuit has risen from the grace », ibid., p. 20.

20. Ibid., p. 17.

21. E. Souchier, op. cit., p. 30.

22. Jérémie Couston, "Midi-Minuit fantastique: histoire d'une légendaire revue des horreurs", Télérama, 18/07/2014, [en ligne], consulté le 21/12/2020, URL : https://www.telerama.fr/cinema/ midi-minuit-fantastique-histoire-d-une-legendaire-revue-des-horreurs,115030.php.

23. É. Losfeld, op. cit., p. 149.

24. Losfeld le décrit comme un «individu assez pittoresque, et, en tout cas, extrêmement drôle, quoique fatigant » (ibid., p. 148).

25. Id.

26. Terme qu'utilise fréquemment N. Stanzick : voir par exemple dans FAL, " "Midi-Minuit fantastique" : l'intégrale de la revue, entretien avec Nicolas Stanzick», lintern@ute, 4/04/2014, [en ligne], consulté le 21/12/2020, URL : http://salon-litteraire.linternaute.com/fr/interviews/ content/1872584-midi-minuit-fantastique-l-integrale-de-la-revue-entretien-avec-nicolas-

stanzick.

27. Jean-Paul Török, « Le maciste ne passera pas », MMF, op. cit., vol. 1, p.. 652.

28. N. Stanzick, ibid., p. 19.

29. É. Losfeld, op. cit., p. 150.

30. M.-È. Thérenty, op. cit., p. 49-123.

31. On peut d'ailleurs considérer que $M M F$ cherche aussi par des moyens détournés à se présenter comme une revue d'art. Cette impression est savamment entretenue par le numéro 2, très marqué par la présence du peintre Félix Labisse.

32. J. Boullet, "King Kong et le Pirée. Quelques fausses illustrations de trucages ", MMF, op. cit., vol. 1, p. 324.

33. M. Caen « Érotisme et sadisme dans l'œuvre de Terence Fisher », MMF, op. cit., vol. 1, p. 52. Par ailleurs, MMF reproduit volontiers des extraits de périodiques anciens. Voir par exemple ce texte traitant des collectionneurs d'autographes d'assassins et autres cordes de pendus, initialement paru en 1906 dans Lectures pour tous (« À propos des musées noirs », MMF, op. cit., vol. 2, p. 258).

34. MMF, op. cit., vol. 1, p. 249.

35. « La fée rageuse », MMF, ibid., p. 407.

36. Voir la description en tête du chapitre XII du conte Ourson.

37. J. Boullet, « Terence Fisher et la permanence des mythes », MMF, op. cit., vol. 1, p. 46.

38. N. Stanzick, ibid., p. 20.

39. Id.

40. M.-È. Thérenty, op. cit., p. 78.

41. Il n'existe pas actuellement de définition parfaitement close de la revue et du magazine. Les deux termes peuvent être utilisés de façon interchangeable mais, de manière générale, il semblerait que le public visé par les revues soit plus pointu que celui visé par les magazines. L'équipe des ressources numériques de l'ENSSIB consacre un point à cette question, et nous faisons nôtres les conclusions de l'auteur'e (malheureusement anonyme) de cet excellent point 
définitionnel : « un magazine serait plus grand public tandis qu'une revue s'adresserait plus à un public de spécialistes ou correspondrait à une publication plus pointue. Ainsi les revues généralistes de réflexion et de débat ou les revues de la recherche en sciences humaines et sociales ne pourraient pas se ranger sous l'appellation "magazine". Une revue est donc une publication sur de grands sujets de réflexion ou d'actualité ou alors elle traite de sujets très spécialisés. Les articles qui la composent sont plus pointus et plus longs que ceux d'un magazine. Une revue peut être illustrée ou non tandis qu'un magazine l'est le plus souvent. » Anonyme, «Différence magazine et revue », Enssib [En ligne], mis en ligne le 10/04/2014, consulté le 04/06/2021, URL : https://www.enssib.fr/services-et-ressources/questions-reponses/differencemagazine-et-revue.

42. MMF, op. cit., vol. 2, p. 282.

43. M. Caen, "Midi-Minuit historique », MMF, op. cit., vol. 2, p. 398.

44. Ibid., p. 399.

45. S. du Mesnildot, op. cit., p. 88.

46. M. Caen, « Midi-Minuit historique », MMF, op. cit., vol. 2, p. 399.

47. M.-È. Thérenty, op. cit., p. 99.

48. É. Losfeld, op. cit., p. 150

49. N. Stanzick, MMF, op. cit., vol. 1, p. 24.

50. Michel Caen, « Midi-Minuit fantastique », MMF, op. cit., vol. 3, p. 331.

51. Voir Sylvain Lesage, « Barbarella : révolution sexuelle ou révolution éditoriale? », dans Alexis Lévrier et Guillaume Pinson (dir.), Les Petits Aventuriers du quotidien. Bande dessinée, journal et imaginaires médiatiques (XIX ${ }^{e}-\mathrm{XXI}{ }^{e}$ siècles), Bruxelles, Les Impressions nouvelles, 2021, p. 127-147.

52. «Le Courrier des lecteurs », MMF, $n^{\circ}$ 20, p. 74-75.

53. MMF publie d'ailleurs une lettre de lui dans le numéro 9.

54. Jean-Pierre Bouyxou, «Cinéma Midi-Minuit? Fantastique!», Siné Mensuel, $1^{\text {er }}$ septembre 2013.

55. Michel Caen et Jean-Claude Romer, « Le studio de l'Étoile », MMF, n 21, avril 1970, p. 2.

56. Michel Caen, « Scialitique... Caméra... Moteur ! ", MMF, op. cit., vol. 2, p. 672.

57. Voir la réclame parue à la toute fin de $M M F, \mathrm{n}^{\circ} 17$, pour l'ouvrage de son collaborateur Jacques Sternberg, Une succursale du fantastique nommée science-fiction.

58. «Lettre de Christopher Lee ", MMF, op. cit., vol. 1, p. 516 : dans sa présentation de la réédition, Nicolas Stanzick précise que, bien entendu, l'acteur répond en fait à une sollicitation de la revue (ibid., p. 13).

59. « Rectificatifs », ibid., p. 596.

60. «Petit courrier des lecteurs », MMF, op. cit., vol. 3, p. 78.

61. « Le courrier des lecteurs", ibid., p. 415.

62. MMF, op. cit., vol. 2, p. 601.

63. M. Caen et J.-C. Romer, « Entretien avec Riccardo Freda », ibid., p. 19.

64. Ibid., p. 22.

65. Bernard Eisenschitz et Jean-Claude Romer, «Entretien avec Edgar G. Ulmer », MMF, op. cit., vol. 3, p. 153.

66. Chris Wicking et Vincent Porter, "Entretien avec Roger Corman ", MMF, op. cit., vol. 2, p. 646.

67. Bertrand Tavernier, « Entretien avec Terence Fisher », ibid., p. 29.

68. Michel Caen, « Entretien avec Terence Fisher », ibid., p. 591.

69. Chris Wicking, « Entretien avec Jacques Tourneur », MMF, op. cit., vol. 3, p. 28.

70. Voir les réactions des journalistes face à l'objet $M M F$ lors de sa réédition, dans cette revue de presse aussi excellente qu'utile, Midi-Minuit fantastique [En ligne], consulté le 29/08/2021, URL : https://midiminuitfantastique.com/presse/.

71. Michel Caen, « Notes de lectures », MMF, op. cit., vol. 3, p. 710. 
72. Michel Caen, «Érotisme et sadisme dans l'œuvre de Terence Fisher », MMF, op. cit., vol. 1, p. 52.

73. Le numéro 17 propose ainsi la «fiche signalétique» de Barbara Steele, mensurations comprises. Les couvertures en couleurs mettent l'accent sur une iconographie agressivement érotique (le numéro 21 et sa femme dépoitraillée en contre-plongée) ou sur un bis frôlant la parodie pornographique (le numéro 22 et ses femmes singes très velues et très fardées).

74. Éric Losfeld redoute quant à lui que $M M F$ ne cède à la «tentation du fascisme » et à la «tentation de l'homosexualité » (Losfeld, op. cit., p. 151). L'équivalence des formules est pour le moins bizarre.

75. S. du Mesnildot, op. cit., p. 82.

76. The Mask of Fu Manchu, de Charles Brabin (1935), est aussi connu sous le titre Le Masque d'or.

77. É. Losfeld, op. cit., p. 150.

78. S. du Mesnildot, op. cit., p. 82.

79. MMF, op. cit., vol. 1, p. 97-106.

80. Ibid., p. 161-168.

81. Ibid., vol. 3, p. 606-636.

82. Voir par exemple les longues pages de filmographies chères à Jean-Claude Romer, ou les longs synopsis auxquels succèdent parfois quelques courtes lignes de critique.

83. N. Stanzick, MMF, op. cit., vol. 1, p. 18.

84. S. du Mesnildot, op. cit., p. 84.

85. Diverses allusions sont faites au fil des interviews de N. Stanzick et M. Caen.

86. Jean-Pierre Putters, Mad... ma vie !, Pertuis, Rouge Profond, 2012, p. 36-37.

87. « Ennio Morricone par Christophe Gans », podcast On aura tout vu, France Inter, 22 mars 2014, [en ligne], URL : https://www.franceinter.fr/emissions/aura-tout-vu/aura-tout-vu-22-mars-2014. L'extrait portant sur MMF est consultable sur Midi-Minuit fantastique [en ligne], URL: http:// midiminuitfantastique.com/wp-content/uploads/2014/03/On-aura-tout-

vu\%28FranceInter22-03-2014\%29MMF-Christophe-Gans.mp3.

\section{RÉSUMÉS}

Premier périodique européen consacré au cinéma de genre, Midi-Minuit fantastique (1962-1970) débute sur le mode de la contestation en dénonçant le goût de la presse généraliste et de la critique cinématographique pour des œuvres jugées bourgeoises. Très hétérogène, cet objet médiatique s'appuie sur une équipe rédactionnelle inexpérimentée, un public mal identifié mais prolixe, un éditeur-libraire avant-gardiste, des réalisateurs/acteurs/studios étrangers (italiens, britanniques et américains surtout), et une production cinématographique internationale souvent invisible. Cette étude montre que Midi-Minuit fantastique est à la fois une revue littéraire, une revue d'art, un fanzine - avant l'heure - déguisé en magazine, un recueil d'images rares comparable aux keepsakes du XIXe siècle et une revue savante balbutiante mais érudite. En superposant ces diverses approches, Midi-Minuit fantastique établit les premières relations entre pays européens et Etats-Unis (principalement), tout en organisant la réception du cinéma, de la littérature et de la BD de genre en France. Rendant visibles pour la première fois des images jusqu'alors introuvables et les entourant de textes tour à tour poétiques, polémiques et érudits, Midi-Minuit fantastique a donc directement modelé les représentations et les fantasmes du public 
français tout en considérant le cinéma de genre en tant que produit industriel, international, populaire ET artistique.

\section{INDEX}

Mots-clés : presse, cinéma, fantastique, horreur, critique cinématographique, cinéma de genre

\section{AUTEUR}

\section{AXEL HOHNSBEIN}

Axel Hohnsbein est maître de conférences à l'université de Bordeaux (EA 4574 - Sciences, Philosophie, Humanités). II travaille principalement sur la vulgarisation scientifique et photographique du XIXe siècle, sur la relation texte/image, les dispositifs optiques et la poétique propre à ces pratiques.

Dernières publications : co-direction avec Bénédicte Jarrasse d'Arts et savoirs $\mathrm{n}^{\circ} 16$ : « Des corps dans la ville. Norme et écart au XIXe siècle », 2021. Publication du livre La Science en mouvement. La presse de vulgarisation scientifique au prisme des dispositifs optiques (1851-1903), Epistémocritique, 2021. 